Revista de Matemática: Teoría y Aplichciones 3(1): 35-44 (1996)

\title{
LA ECUACIÓN DE HILL CON POTENCIAL IRREGULAR
}

\author{
Santiago Cambronero Villalobos ${ }^{1}$
}

\begin{abstract}
Resumen
Se considera la ecuación de Hill cuyo potencial es la derivada formal de una función Hölder - continua de parámetro $\theta \in(0,1)$, y se muestra que las soluciones de la versión discreta correspondiente convergen adecuadamente a las soluciones de la ecuación original. Este hecho se usa para establecer teoremas de existencia de soluciones para este caso singular, y para deducir algunas propiedades de las soluciones y el discriminante de la ecuación estudiada.
\end{abstract}

\begin{abstract}
We consider the Hill equation whose potential is the formal derivative of a Hölder - continuous function of parameter $\theta \in(0,1)$, and show that solutions of the discrete version converge to solutions of the original equation in a suitable way. This fact is used to establish existence and uniqueness theorems for this singular case, and to deduce some properties of solutions and the discriminant of the studied equation.
\end{abstract}

\section{Introducción}

La ecuación de Hill con potencial $Q$ está dada por

$$
-y^{\prime \prime}+Q y=\lambda y
$$

con $\lambda \in$ C. La mayoría de los resultados existentes hasta el momento acerca de esta ecuación asumen que $Q$ es suave. En [1] y [2] se trata el caso de potenciales singulares aleatorios, como es el caso de "ruido blanco". Sin embargo, el lector requiere de ciertos conocimientos en probailidad para entender dichos trabajos. En el presente trabajo se retoman algunos de los métodos usados en las referencias antes citadas y se adaptan al caso de un potencial no aleatorio, con el fin simplificar el tratamiento y a la vez generalizar los resultados a potenciales menos regulares.

Se considera la versión discreta de (1):

$$
-\Delta^{2} u_{i}+Q_{i} u_{i}=\lambda u_{i}, \quad \text { para } i=0,1, \ldots
$$

donde $u_{i}^{\prime}=n\left(u_{i+1}-u_{i}\right), \Delta^{2} u_{i}=n^{2}\left(u_{i+1}-2 u_{i}+u_{i-1}\right)$, y se demuestra que las soluciones de (2) convergen a las de (1) en una manera adecuada, lo que servirá para deducir una serie de propiedades acerca de las soluciones de (1).

\footnotetext{
${ }^{1}$ Escuela de Matemática, Universidad de Costa Rica, 2060 San José, Costa Rica
} 
Denotaremos por $-\Delta^{2}+Q$ al operador definido por el lado izquierdo de (2). Éste es un operador definido en el conjunto de sucesiones complejas. La sucesión $\left(u_{i}\right)_{i \geq 0}$ se llama periódica si $u_{i+n}=u_{i}, \forall i \in \mathbf{N}$. Diremos que $\lambda \in \mathbf{C}$ es un valor propio periódico de $-\Delta^{2}+Q$, si existe una solución periódica no nula de (2).

\section{Algunos Preliminares}

Para $Q=\left(Q_{0}, \ldots, Q_{n-1}\right), u=\left(u_{0}, \ldots, u_{n-1}\right), v=\left(v_{0}, \ldots, v_{n-1}\right) \in \mathbf{C}^{n}$ definamos

$$
\begin{aligned}
\mathcal{D}(u, v) & =\sum_{i=0}^{n-1} u_{i}^{\prime} \bar{v}_{i}^{\prime} h+\sum_{i=0}^{n-1} Q_{i} u_{i} \bar{v}_{i} h \\
\mathcal{H}(u, v) & =\sum_{i=0}^{n-1} u_{i} \bar{v}_{i} h
\end{aligned}
$$

con $h=1 / n$.

Definimos $Q_{i+n}=Q_{i}$, y escribimos $b_{0}=0, b_{i}=h\left(Q_{0}+\ldots+Q_{i-1}\right)$,

$$
|b|=\max \left\{\left|b_{i}\right|: i=1, \ldots, n\right\} .
$$

El siguiente lema será de gran utilidad en el presente trabajo.

Lema $1 \lambda_{0}(Q)=\min \left\{\mathcal{D}(u, u): u_{n}=u_{0}, \mathcal{H}(u, u)=1\right\}$ existe para todo $Q \in \mathbf{R}^{n}, y$ $\lambda_{0}(Q) \geq-|b|(|b|+1)$.

\section{PRUEBA:}

Por compacidad del conjunto $\left\{u: \sum u_{i}^{2} h=1\right\}$ en $\mathbf{R}^{n}$, el mínimo existe. Ahora si $\mathcal{H}(u, u)=$ 1 , entoces $\left|u_{i}\right| \leq 1$ para algún $i$. Supongamos entonces $\left|u_{0}\right| \leq 1$, para simplificar. Sumando por partes tenemos

$$
\mathcal{D}(u, u)=\sum\left|u_{i}^{\prime}\right|^{2} h+b_{n}\left|u_{n}\right|^{2}-\sum b_{i+1}\left(\left|u_{i+1}\right|^{2}-\left|u_{i}\right|^{2}\right) .
$$

Tomando $T=\left(\sum\left|u_{i}^{\prime}\right|^{2} h\right)^{\frac{1}{2}}$, la desigualdad de Shwarz's implica

$$
\left.\sum|| u_{i+1}\right|^{2}-\left|u_{i}\right|^{2} \mid \leq 2\left(\sum\left|u_{i}\right|^{2} h\right)^{\frac{1}{2}}\left(\sum\left|u_{i}^{\prime}\right|^{2} h\right)^{\frac{1}{2}}=2 T,
$$

y entonces

$$
\mathcal{D}(u, u) \geq T^{2}-|b|(1+2 T)=(T-|b|)^{2}-|b|(|b|+1),
$$

en vista de $\left|u_{n}\right|=\left|u_{0}\right| \leq 1$.

Observación: Note que si $\mathcal{H}(u, u)=1$ y $v_{i}=\left|u_{i}\right|$, entonces $\mathcal{H}(v, v)=1,\left|v_{i}^{\prime}\right| \leq\left|u_{i}^{\prime}\right|$, y como consecuencia tenemos $\mathcal{D}(v, v) \leq \mathcal{D}(u, u)$. Esto es, si $\mathcal{D}(u, u)=\lambda_{0}(Q)$, $u$ se puede tomar no negativo. Pero ahora, se sigue de $(2)$ que $u_{i}>0$ para todo $i$. Esto se va a asumir en lo que sigue sin más comentario. 
Lema 2 Sea $\lambda_{0}(Q)=\mathcal{D}(u, u)$ y extienda $u$ a todo $\mathbf{N}$ por $u_{i+n}=u_{i}$. Entonces $u$ es una solución periódica de (2) con $\lambda=\lambda_{0}(Q)$. i.e. $\lambda_{0}(Q)$ es un valor propio periódico del operador $-\Delta^{2}+Q$.

\section{PRUEBA:}

Sea $v$ real y tal que $v_{n+i}=v_{i}$ para todo $i$. Entonces $\mathcal{D}(u+\varepsilon v, u+\varepsilon v) / \mathcal{H}(u+\varepsilon v, u+\varepsilon v)$ tiene un mínimo local en $\varepsilon=0$, y consecuentemente $\mathcal{D}(u, v)=\lambda_{0} \mathcal{H}(u, v)$. Pero $\sum u_{i}^{\prime} v_{i}^{\prime} h=$ $-\sum\left(\Delta^{2} u_{i}\right) v_{i} h$, y entonces $\sum\left(-\Delta^{2} u_{i}+Q_{i} u_{i}-\lambda_{0} u_{i}\right) v_{i} h=0$ para cualquier $v$ de esos. Entonces $u$ satisface (2).

Lema 3 Todos los valores propios periódicos de $-\Delta^{2}+Q$ son reales, y $\lambda_{0}(Q)$ es el más pequeño.

\section{Prueba:}

Si $\lambda$ es un valor propio periódico y $u$ es la solución correspondiente, entonces $\lambda \mathcal{H}(u, u)=$ $\sum\left(-\Delta^{2} u_{i}+Q_{i} u_{i}\right) \bar{u}_{i} h=\mathcal{D}(u, u)$. Luego $\lambda$ es real y $\lambda \geq \lambda_{0}(Q)$.

\section{La versión continua}

Consideremos ahora una función $b:[0, \infty[\rightarrow \mathbf{R}$, la cual es Hölder-continua con parámetro $\theta$, o sea,

$$
|b(t)-b(s)| \leq M|t-s|^{\theta}, \text { para } t, s \geq 0, \text { donde } 0<\theta<1 .
$$

Asuma además que $b(0)=0$, y que

$$
b(t+1)-b(1)=b(t), \text { para } 0 \leq t<1 .
$$

Considere la ecuación (1) cuyo potencial $Q$ es la derivada formal de $b$. Más precisamente, la ecuación (1) se interpreta como

$$
-y^{\prime}(x)+y^{\prime}(0)+\int_{0}^{x} y(t) Q(t) d t=\lambda \int_{0}^{x} y(t) d t
$$

donde la intergral en el lado izquierdo representa

$$
y(x) b(x)-\int_{0}^{x} b(t) y^{\prime}(t) d t
$$

Nótese que $Q$ no existe en el sentido clásico, a menos que $b$ sea derivable. La condición (4)

dice que $Q$ es periódica de período 1. Defina $Q_{i}$ como $n\left(b_{i+1}-b_{i}\right)$, donde $b_{i}=b\left(\frac{i}{n}\right)$. En lo que sigue, consideramos (2) con potencial $Q^{(n)}=\left(Q_{0}, \ldots, Q_{n-1}\right)$ y escribimos $\lambda^{(n)}=$ $\lambda_{0}\left(Q^{(n)}\right)$. Vamos a mostrar más adelante que la sucesión $\left(\lambda^{(n)}\right)$ converge al primer valor propio de (1). De ahora en adelante, $|b|$ denotará la norma sup de $b(t)$ en $[0,1]$. 


\section{Condiciones Iniciales}

Fijemos $\lambda \in \mathbf{C}$ y consideremos una solución de (2) con condiones iniciales fijas $u_{0}=\alpha$, $u_{0}^{\prime}=\beta$ independentes de $n$. Vamos a mostrar que dicha solución converge a la solución correspondiente de (1).

Note primero que de (2) se tiene

$$
\begin{aligned}
u_{j}^{\prime}-u_{k}^{\prime}= & \sum_{i=k+1}^{j}\left(Q_{i}-\lambda\right) u_{i} h \\
= & \sum_{i=k+1}^{j}\left(Q_{i}-\lambda\right) h\left[u_{0}+\sum_{l=0}^{i-1} u_{l}^{\prime} h\right] \\
= & u_{0} \sum_{i=k+1}^{j}\left(Q_{i}-\lambda\right) h+\sum_{l=0}^{k} u_{l}^{\prime} h \sum_{i=k+1}^{j}\left(Q_{i}-\lambda\right) h \\
& +\sum_{l=k+1}^{j-1} u_{l}^{\prime} h \sum_{i=l+1}^{j}\left(Q_{i}-\lambda\right) h,
\end{aligned}
$$

y que

$$
\left|\sum_{i=l+1}^{j}\left(Q_{i}-\lambda\right) h\right|=\left|b_{j+1}-b_{l+1}-\lambda(j-l) h\right| \leq C \varepsilon^{\theta}
$$

para $k \leq l \leq j-1$, siempre que $(j-k) h \leq \varepsilon \leq 1$. Aquí $C$ depende sólo de $b$, siempre que $\lambda$ se mantenga acotado. Luego,

$$
\left|u_{j}^{\prime}-u_{k}^{\prime}\right| \leq C \varepsilon^{\theta}\left[|\alpha|+\sum_{l=0}^{j-1}\left|u_{l}^{\prime}\right| h\right]
$$

En particular, para $k=0$,

$$
\left|u_{j}^{\prime}\right| \leq c_{1}+C \sum_{l=0}^{j-1}\left|u_{l}^{\prime}\right| h
$$

con $c_{1}=|\beta|+C|\alpha|$. Se sigue por inducción que

$$
\left|u_{j}^{\prime}\right| \leq c_{1}(1+C h)^{j} \leq c_{1}(1+C h)^{n} \leq c_{1} e^{C} .
$$

i.e. $\left|u_{j}^{\prime}\right|$ es uniformemente acotada. Ahora (5) implica

$$
\left|u_{j}^{\prime}-u_{k}^{\prime}\right| \leq C \varepsilon^{\theta}
$$

con una nueva constante $C$.

Denote por $\psi_{n}$ el camino poligonal determindo por los valores $u_{j}$ en los puntos $j / n, \mathrm{y}$ por $\hat{\psi}_{n}$ el camino correspondiente a los valores $u_{j}^{\prime}$. Entonces $\left(\psi_{n}\right)$ y $\left(\hat{\psi}_{n}\right)$ son equicontinuas 
y uniformemente acotadas. Esto implica que existe una subsucesión $\left(\psi_{n_{k}}\right)$ que converge uniformemente a una función continua $\psi$, y tal que $\left(\hat{\psi}_{n_{k}}\right)$ converge uniformemente a una función continua $\widehat{\psi}$. Es fácil ver que entonces $\psi$ es derivable, y $\psi^{\prime}=\widehat{\psi}$.

De la identidad ${ }^{2}$

$$
u_{j}^{\prime}=\beta+b_{j+1} u_{j}-\sum_{k=0}^{j-1} b_{k+1} u_{k}^{\prime} h-\lambda \sum_{k=1}^{j} u_{k} h
$$

obtenemos

$$
\psi^{\prime}(x)=\beta+b(x) \psi(x)-\int_{0}^{x} b(t) \psi^{\prime}(t) d t-\lambda \int_{0}^{x} \psi(t) d t .
$$

Además $\psi(0)=\alpha$, y entonces $\psi$ es solución del problema

$$
\begin{aligned}
& -y^{\prime \prime}+Q y=\lambda y \\
& y(0)=\alpha, y^{\prime}(0)=\beta .
\end{aligned}
$$

Esto muestra en particular la existencia de soluciones de (6); la unicidad se muestra abajo.

Teorema 1 Si $Q=b^{\prime}$, donde $b$ es Hölder continua de parámetro $\left.\theta \in\right] 0,1[$, entonces (6) tiene una solución única para todo $\alpha, \beta$ y $\lambda \in \mathbf{C}$.

PRUEBA:

Sea $\psi$ solución de (6) con $\alpha=\beta=0$, que significa

$$
\psi^{\prime}(x)=b(x) \psi(x)-\int_{0}^{x} b(t) \psi^{\prime}(t) d t-\lambda \int_{0}^{x} \psi(t) d t
$$

de donde

$$
\left|\psi^{\prime}(x)\right| \leq|b||\psi(x)|+|b| \int_{0}^{x}\left|\psi^{\prime}(t)\right| d t+|\lambda| \int_{0}^{x}|\psi(t)| d t
$$

el resto es rutina.

La unicidad de $\psi$ implica que realmente la sucesión $\psi_{n}$ converge a $\psi$, pues para toda subsucesión de $\left(\psi_{n}\right)$, hay siempre una sub-subsucesión que converge a $\psi$. Ahora, como $\left(\psi_{n}\right)$ es localmente acotada en $\lambda$, se sigue que $\psi$ es entera como funcción de $\lambda$. Para resumir:

Teorema 2 Considere la solución $\left(u_{0}, u_{1}, \ldots\right)$ de (2), con $u_{0}=\alpha$ y $u_{0}^{\prime}=\beta$. Los correspondientes caminos poligonales $\psi_{n}$ y $\hat{\psi}_{n}$ convergen uniformemente a $\psi$ y $\psi^{\prime}$ respectivamente, en el intervalo $[0,1]$, con $\psi$ la solución of (6). Esta solución es una función entera de $\lambda$.

\footnotetext{
${ }^{2}$ Compare con (2) y use suma parcial.
} 
Una pequeña modificación a la prueba del teorema 2 muestra el siguiente corolario.

Corolario. Si $\left(\lambda_{n}\right)$ es una sucesión de números complejos que converge a algún $\lambda$, entonces la solución $\psi_{n}$ de $(2)$ correspondiente a $\lambda_{n}$, con $\psi_{n}(0)=\alpha$ y $\psi_{n}^{\prime}(0)=\beta$, converge a la solución de (6) en el sentido poligonal empleado arriba.

Notación: De ahora en adelante, $\left(\psi_{j}\right)$ denotará la solución de $(2)$ con $\psi_{0}=1$ y $\psi_{0}^{\prime}=0$, y $\left(\phi_{j}\right)$ la solución con $\phi_{0}=0, \phi_{0}^{\prime}=1$. Las letras $\psi$ y $\phi$ denotarán las soluciones correspondientes de (1). Las soluciones generales serán denotadas por $\varphi_{j}$ y $\varphi$ respectivamente.

\section{Condiciones Cuasi-Periódicas}

Considere el problema de encontrar una solución $\left(\varphi_{j}\right)$ de $(2)$ que satisfaga las condiciones

$$
\varphi_{i+n}=m_{n} \varphi_{i}
$$

para algún $m_{n}>1$.

Toda solución de (2) satisface

$$
\begin{aligned}
\varphi_{j}^{\prime} & =\varphi_{j-1}^{\prime}+\left(Q_{j}-\lambda\right) h \varphi_{j} \\
\varphi_{j+1} & =\left(2+\left(Q_{j}-\lambda\right) h^{2}\right) \varphi_{j}-\varphi_{j-1}
\end{aligned}
$$

de donde es obvio que, para que la solución satisfaga (7) es suficiente que satisfaga

$$
\varphi_{n}=m_{n} \varphi_{0}, \text { y } \varphi_{n}^{\prime}=m_{n} \varphi_{0}^{\prime} .
$$

Escrribiendo $\varphi_{j}=c_{1} \psi_{j}+c_{2} \phi_{j}$, estas condiciones se transforman en

$$
\begin{aligned}
c_{1} \psi_{n}+c_{2} \phi_{n} & =m_{n} c_{1}, \\
c_{1} \psi_{n}^{\prime}+c_{2} \phi_{n}^{\prime} & =m_{n} c_{2} .
\end{aligned}
$$

entonces, $m_{n}$ debe ser un valor propio de la matrix

$$
M=\left[\begin{array}{cc}
\psi_{n} & \phi_{n} \\
\psi_{n}^{\prime} & \phi_{n}^{\prime}
\end{array}\right]
$$

y consecuentemente $m_{n}^{2}-2 \Delta_{n} m_{n}+w_{n}=0 ;$ aquí $\Delta_{n}=\frac{1}{2}\left(\psi_{n}+\phi_{n}^{\prime}\right)$ y

$$
w_{j}=\psi_{j} \phi_{j}^{\prime}-\psi_{j}^{\prime} \phi_{j}=n\left(\psi_{j} \phi_{j+1}-\psi_{j+1} \phi_{j}\right)
$$

Note que $w_{0}=1 \mathrm{y}$, por la segunda identidad en (8), que $w_{j}=w_{j-1}$, para todo $j$. entonces $w_{j} \equiv 1$. En particular $w_{n}=1$. La ecuación $m_{n}^{2}-2 \Delta_{n} m_{n}+1=0$ tiene soluciones reales si y sólo si $\left|\Delta_{n}\right| \geq 1$.

Por otro lado, para que una solución $\varphi=c_{1} \psi+c_{2} \phi$ de (1) satisfaga

$$
\varphi(x+1)=m \varphi(x),
$$


se encuentra de la misma manera que $m$ debe satisfacer

$$
m^{2}-2 \Delta m+w(1)=0
$$

con $\Delta=\frac{1}{2}\left(\psi(1)+\phi^{\prime}(1)\right)$ y $w(t)=\psi(t) \phi^{\prime}(t)-\psi(t)^{\prime} \phi(t)$.

Por el teorema 2, $w(t)=\lim _{n \rightarrow \infty} w_{j}=1($ para $j / n \rightarrow t)$, y entonces $w \equiv 1$. Este hecho se obtiene también del siguiente cálculo formal:

$$
w^{\prime \prime}=\psi \phi^{\prime \prime}-\psi^{\prime \prime} \phi=\psi(Q-\lambda) \phi-\phi(Q-\lambda) \psi=0 .
$$

Por el mismo teorema,

$$
\Delta(\lambda)=\lim _{n \rightarrow \infty} \Delta_{n}(\lambda)
$$

Si se tiene $|\Delta(\lambda)|>1$, se sigue que $\left|\Delta_{n}(\lambda)\right|>1$ para $n$ grande, y consecuentemente

$$
m_{n}=\Delta_{n}+\sqrt{\Delta_{n}^{2}-1} \rightarrow m=\Delta+\sqrt{\Delta^{2}-1}
$$

Ahora podemos establecer el siguiente teorema.

Teorema 3 Suponga $|\Delta(\lambda)|>1$ para algún $\lambda$. Entonces hay una solución $\varphi$ de (1), con $\varphi(x+1)=m \varphi(x)$. Además, para $n$ grande hay una solución $\left(\varphi_{j}\right)$ de (2), $\operatorname{con} \varphi_{i+n}=m_{n} \varphi_{i}$, la cual converge a $\varphi$ en el sentido poligonal.

\section{Prueba:}

Si $\varphi=c_{1} \psi+c_{2} \phi$, entonces

$$
\begin{array}{ll}
c_{1} \psi(1)+c_{2} \phi(1) & =m c_{1} \\
c_{1} \psi^{\prime}(1)+c_{2} \phi^{\prime}(1) & =m c_{2} .
\end{array}
$$

Podemos escoger siempre las soluciones $\left(c_{1}, c_{2}\right)$ de $(9)$ y $(10)$ de tal forma que $c_{1}=1$ o $c_{2}=1$. Entonces, como los coeficientes de (9) convergen a los de (10), las soluciones corespondentes de (9) también convergen a las de (10), y esto es todo lo que se necesita.

El caso $|\Delta|=1$ es más delicado, pues podemos tener $\left|\Delta_{n}\right|<1$ para todo $n$. En lo que sigue tratamos el caso $\lambda=\lambda_{0}(Q)$.

\section{Convergencia del Primer Valor Propio}

Podemos ahora probar la existencia de $\lambda_{0}(Q)$ como el límite de la sucesión $\lambda^{(n)}$ de primeros valores propios de (2). Empezaremos por establecer otros resultados acerca de dicha sucesión.

Lema 4 La sucesión $\left(\lambda^{(n)}\right)$ es acotada. 
Prueba:

Por definición, $\lambda^{(n)} \leq \mathcal{D}(1,1)=b(1) \leq|b|$, y $\left(\lambda^{(n)}\right)$ es también acotada por abajo, por el lema 1.

Lema 5 Asuma que $\lambda$ es un valor propio periódico de (1). Entonces

$$
\limsup _{n \rightarrow \infty} \lambda^{(n)} \leq \lambda
$$

Prueba:

Sea $\varphi$ la solución periódica correspondiente de (1), normalizada para que $\int_{0}^{1} \varphi^{2}=1$, y defina $\varphi_{j}=\varphi(j / n)$, para $j=0,1, \ldots, n$, y cualquier $n$. Tenemos

$$
\lambda^{(n)} \leq\left[\sum_{j=0}^{n-1}\left|\varphi_{j}^{\prime}\right|^{2} h+\sum_{j=0}^{n-1} Q_{j} \varphi_{j}^{2} h\right] /\left(\sum_{j=0}^{n-1} \varphi_{j}^{2} h\right)
$$

Cuando $n \rightarrow \infty$, el lado derecho converge a $\int_{0}^{1}\left(\left|\varphi^{\prime}\right|^{2}+Q \varphi^{2}\right)=\lambda$. La justificación es rutina.

Lema 6 Sea u la solución periódica de (2) correspondiente a $\lambda^{(n)}$, normalizada para que $\sum u_{j}^{2} h=1$, y sea $C_{0}=|b|+\sqrt{|b|^{2}+2|b|}$. Entonces hay un $j$ tal que $\left|u_{j}^{\prime}\right| \leq C_{0}$.

\section{Prueba:}

Suponga $\left|u_{j}^{\prime}\right|>C_{0}$, para $j=0, \ldots, n-1$. Entonces $\sqrt{\sum\left|u_{i}^{\prime}\right|^{2} h}>C_{0}$, y consecuentemente $b(1)=\mathcal{D}(1,1) \geq \mathcal{D}(u, u)>\left[C_{0}-|b|\right]^{2}-|b|(|b|+1)=|b| ;$ compare con la prueba de lemma 1. Esto es una contradicción.

Lema 7 Sea u como en el lema 6. Entonces hay una constante $C$, independiente de $n$, tal que $\left|u_{j}^{\prime}\right| \leq C$ y $\left|u_{j}\right| \leq C$ para $j=0,1, \ldots, n$, y todo $n \geq 1$.

PRUEBA:

Por la periodicidad, podemos asumir $\left|u_{0}^{\prime}\right| \leq C_{0}$. Ahora para $j>k$,

$$
u_{j}^{2}=u_{k}^{2}+\sum_{i=k}^{j-1}\left(u_{i+1}^{2}-u_{i}^{2}\right)
$$

y entonces, como en la prueba del lema 1 ,

$$
u_{j}^{2} \leq u_{k}^{2}+2 T_{n}, \quad \text { con } T_{n}=\left(\sum\left|u_{i}^{\prime}\right|^{2} h\right)^{\frac{1}{2}}
$$

Lo mismo es cierto para $k \geq j$, y entonces tomando el promedio sobre $k$ tenemos

$$
u_{j}^{2} \leq \sum u_{k}^{2} h+2 T_{n}=1+2 T_{n}: \text { for } j=0,1, \ldots, n-1 .
$$


Por otro lado,

$$
\begin{aligned}
u_{j}^{\prime} & =u_{0}^{\prime}+\sum_{i=1}^{j} u_{i}\left(b_{i+1}-b_{i}\right)-\lambda^{(n)} \sum_{i=1}^{j} u_{i} h \\
& =u_{0}^{\prime}+b_{j+1} u_{j}-b_{1} u_{0}-\sum_{i=1}^{j} b_{i}\left(u_{i}-u_{i-1}\right)-\lambda^{(n)} \sum_{i=1}^{j} u_{i} h
\end{aligned}
$$

de donde

$$
\left|u_{j}^{\prime}\right| \leq K_{n}+|b| \sum_{i=0}^{j-1}\left|u_{i}^{\prime}\right| h,
$$

con $K_{n}=\left|\lambda^{(n)}\right|+C_{0}+2|b|\left(1+2 T_{n}\right)^{\frac{1}{2}}$, y por inducción sobre $j$ se sigue que

$$
\left|u_{j}^{\prime}\right| \leq K_{n}(1+|b| h)^{j} \leq K_{n} e^{|b|}, \text { for } j=0,1, \ldots, n-1 .
$$

Entonces $T_{n} \leq K_{n} e^{|b|}$, y como lasucesión $\left(\lambda^{(n)}\right)$ es acotada, esto implica que $\left(T_{n}\right)$ y $\left(K_{n}\right)$ son acotadas también.

Denotemos por $\psi_{n}$ el camino poligonal determinado por los valores $u_{j}$ en los puntos $j / n$, donde $u$ es dado por el lema 6 . Entonces $\left(\psi_{n}\right)$ es equicontinua y uniformemente acotada. Tenemos también acotación uniforme de la sucesión $\hat{\psi}_{n}$ determinada por los valores $u_{j}^{\prime}$ en los puntos $j / n$. La equicontinuidad de esta sucesión es obtenida en la misma forma que en el caso de condiciones iniciales. Nótese que ahora no tenemos $\lambda$ fijo, pero como $\left(\lambda^{(n)}\right)$ es acotada, los detalles son básicamente los mismos.

Dada una subsucesión $\left(\lambda^{\left(n_{k}\right)}\right)$ que converge a algún $\lambda$, podemos escoger una subsubsucesión (que denotaremos en la misma forma), tal que $\left(\psi_{n_{k}}\right)$ converge uniformemente a una una función $\psi$ de clase $\mathcal{C}^{1}, \mathrm{y}\left(\widehat{\psi}_{n_{k}}\right)$ converge uniformente a $\psi^{\prime}$. Se sigue que $\psi$ es una solución de (2), y que $\int_{0}^{1} \psi=1$.

Combinando esto con el lema 5 , obtenemos que cualquier subsucesión convergente de $\left(\lambda^{(n)}\right)$ debe converger a $\lim \sup \lambda^{(n)}$. Esto muestra lo siguiente.

Teorema 4 La sucesión $\left(\lambda^{(n)}\right)$ converge a $\lambda_{0}$. i.e.

$$
\lim _{n \rightarrow \infty} \lambda_{0}\left(Q^{(n)}\right)=\lambda_{0}(Q)
$$

Además, si $\left(u_{0}, u_{1}, \ldots\right)$ es la solución periódica de (2) correspondiente a $\lambda^{(n)}$, y $\psi$ es la solución periódica de (1) correspondiente a $\lambda_{0}(Q)$, entonces $\left(u_{j}\right)$ converge a $\psi$ en el sentido poligonal.

\section{Observaciones:}

Los resultados establecidos hasta el momento se pueden usar para probar una serie de propiedades de la ecuación de Hill, probando primero sus versiones discretas. Por ejemplo, la solución correspondiente al primer valor propio de (2) se puede tomar positiva; entonces, 
por el teorema anterior, lo mismo es cierto para la solución de (1) correspondiente al primer valor propio $\lambda_{0}(Q)$.

Se puede observar del lema 5 que $\lambda_{0}$ es una cota inferior del conjunto

$$
\left\{\int_{0}^{1}\left(\varphi^{\prime}+Q \varphi^{2}\right): \varphi \in \mathcal{C}^{1}\left(S^{1}\right) \operatorname{con} \int_{0}^{1} \varphi^{2}=1\right\}
$$

y por otro lado, por el teorema anterior, $\lambda_{0}$ pertenece a dicho conjunto. Esto es

$$
\lambda_{0}(Q)=\min \left\{\int_{0}^{1}\left(\varphi^{\prime}+Q \varphi^{2}\right): \varphi \in \mathcal{C}^{1}\left(S^{1}\right) \operatorname{con} \int_{0}^{1} \varphi^{2}=1\right\},
$$

Esta clase de argumento será aplicado en lo que sigue para estudiar el discriminante de $\Delta(\lambda)$ de $(1)$

\section{Propiedades del Discriminante}

Teorema $5 \Delta(\lambda)$ es una función entera de $\lambda$, de orden $\leq \frac{1}{2}$. Además, para $\lambda<\lambda_{0}(Q)$ tenemos $\Delta(\lambda)>1$.

Prueba:

De (8) se sigue que $\psi_{j} \mathrm{y} \phi_{j}^{\prime}$ son polinomios en $\lambda$, cuyos términos de mayor grado son $h^{2(j-1)}(-\lambda)^{j-1}$ y $h^{2 j}(-\lambda)^{j}$ respectivamente. Entonces $\Delta_{n}$ es un polinomio en $\lambda$, con término de mayor grado $\frac{1}{2} h^{2 n}(-\lambda)^{n}$.

Consecuentemente, $\Delta_{n}(\lambda) \rightarrow+\infty$, cuando $\lambda \rightarrow-\infty$. Ahora como $\Delta_{n} \neq 1$ para $\lambda<\lambda_{n}$, se sigue por continuidad que $\Delta_{n}(\lambda)>1$, para estos valores de $\lambda$. Dado $\lambda<\lambda_{0}(Q)$, el teorema anterior implica $\lambda<\lambda^{(n)}$ para valores grandes de $n$, y consecuentemente $\Delta(\lambda)=\lim \Delta_{n}(\lambda) \geq 1$. Pero la igualdad no se da aquí, pues $\lambda<\lambda_{0}(Q)$. Esto prueba la última afirmación del enunciado.

Que $\Delta(\lambda)$ es una función entera se sigue del teorema 2.

Finalmente, si $|b| \leq c$, no es difícil ver que $\psi$ y $\phi$ son mayorizadas por la solución $w$ de

$$
\begin{aligned}
& w^{\prime \prime}=2 c w^{\prime}+|\lambda| w \\
& w(0)=1, w^{\prime}(0)=1,
\end{aligned}
$$

de donde se sigue la afirmación acerca del orden de $\Delta$.

Un corolario del teorema 5 es que $\Delta(\lambda)$ es localmente acotada en $\lambda$ y $|b|$, i.e. $|\Delta(\lambda)| \leq C$ si $|\lambda| \leq c$ y $|b| \leq c$, donde $C$ depende sólo de $c$. Lo mismo es cierto para $\dot{\Delta}(\lambda)=\frac{d}{d \lambda} \Delta(\lambda)$ y $\ddot{\Delta}(\lambda)$. Por ejemplo

$$
\dot{\psi}(x, \lambda)=\int_{0}^{x}[\psi(x) \phi(t)-\psi(t) \phi(x)] \psi(t) d t
$$

y similarmente para $\dot{\psi}^{\prime}(\cdot, \lambda)$, y así sucesivamente. En resumen:

Corolario. Para $|b| \leq c$ y $|\lambda| \leq c$, hay una constante $C$, que depende sólo de $c$, tal que

$$
\max \{|\Delta(\lambda)|,|\dot{\Delta}(\lambda)|,|\ddot{\Delta}(\lambda)|\} \leq C .
$$




\section{Referencias}

[1] Cambronero, S. \& McKean, H.P. (1995) "Valores propios de Dirichlet asociados a la ecuación de Hill con potencial de ruido blanco", Revista de Matemática: Teoría y Aplicaciones 2(2): 1-7.

[2] Cambronero, S. (1996) The Distribution of the Ground State of Hill's Equation with Random Potential. Tesis de Doctorado. Courant Institute of Mathematical Sciences, New York University.

[3] Eastham, M.S.P. (1973) The Spectral Theory of Periodic Differential Equations. Scottish Academic Press.

[4] Frish, H.L. \& Lloyd, S.P. (1960) "Electron levels in a one-dimensional random lattice", Phys. Review., 120, No. 4: 1175-1189.

[5] Fukushima, M. \& Nakao, S. (1977) "On spectra of the Schrödinger operator with a white noise potential", Z. Wahrsch. Verb. Geb., 37: 267-274.

[6] Halperin, B.I. (1965) "Green's functions for a particle in a one-dimensional potential", Phys. Review., 139, No. 1A: 104-117.

[7] Hochstadt, H. (1961) "Asymptotic estimates for the Sturm - Liouville spectrum", Com. Pure Appl., 14: 749-764.

[8] Hochstadt, H. (1963) "Function-theoretic properties of the discriminant of Hill's equation", Math. Zeit., 82: 237-242.

[9] Kelley, W.G. \& Peterson, S.C. (1991) Difference Equations. Academic Press, Boston.

[10] Levitan, B.M. \& Sargsjar, I.S. (1991) Sturm Liouville and Dirac Operators. Kluwer Academic Publishers.

[11] Lifshits, I.M.; Gradeskul, S.A. \& Pastur, L.A. (1988) Introduction to the Theory of Disordered Systems. John Wiley \& Sons, New York.

[12] Magnus, W. \& S. Winkler, S. (1966) Hill's Equation. Interscience. 